\title{
Parameter uniform numerical methods for singularly perturbed delay parabolic differential equations with non-local boundary condition
}

\author{
Wakjira Tolassa Gobena ${ }^{1}$, Gemechis File Duressa ${ }^{2}$ \\ ${ }^{1 * 2}$ Department of Mathematics, Jimma University, Jimma,P.O.Box378, ETHIOPIA \\ *Corresponding Author: e-mail: wakjira.tolassa@gmail.com, Tel +251-913349537, \\ ORCID iD:http://orcid.org/0000-0002-8885-5264 (Gobena)
}

\begin{abstract}
The motive of this paper is, to develop accurate and parameter uniform numerical method for solving singularly perturbed delay parabolic differential equation with non-local boundary condition exhibiting parabolic boundary layers. Also, the delay term that occurs in the space variable gives rise to interior layer. Fitted operator finite difference method on uniform mesh that uses the procedures of method of line for spatial discretization and backward Euler method for the resulting system of initial value problems in temporal direction is considered. To treat the non-local boundary condition, Simpsons rule is applied. The stability and parameter uniform convergence for the proposed method are proved. To validate the applicability of the scheme, numerical examples are presented and solved for different values of the perturbation parameter. The method is shown to be accurate of $O\left(h^{2}+\Delta t\right)$. Finally, conclusion of the work is included at the end.
\end{abstract}

Keywords: Delay parabolic differential equation, Singular perturbation problem, non-local boundary condition, non-standard finite difference, uniformly convergent.

DOI: http://dx.doi.org/10.4314/ijest.v13i2.7

Cite this article as:

Gobena W.T., Duressa G.F. 2021. Parameter uniform numerical methods for singularly perturbed delay parabolic differential equations with non-local boundary condition. International Journal of Engineering, Science and Technology, Vol. 13, No. 2, pp. 57-71. doi: 10.4314/ijest.v13i2.7

Received: April 25, 2021; Accepted: May 17, 2021; Final acceptance in revised form: June 7, 2021

\section{Introduction}

Over the last few years, the development of numerical methods for the solution of singularly perturbed delay differential equations with non-local boundary conditions has been an important research topic in the mathematical modeling of: epidemiology and population dynamics (Kuang, 1993), physiological kinetics (Baker et al., 1999), production of blood cell (Mahaffy et al.,1999) etc., that take in to consideration the effect of present situation as well as the past history of the physical system. As the characteristics problem when perturbation parameter $\mathcal{E}$ are parallel to the boundary of the domain parabolic layers exhibit in the solution. Also, the delay term that occurs in the space variable gives rise to interior layers.

Most of the traditional numerical methods are not suitable for solving singularly perturbed problems (SPPs) because the presence of the perturbation parameter $\mathcal{E}$, leads to occurrences of divergence in the computed solution (Kumar and Kadalbajoo, 2011). To overcome these divergence, a large number of mesh points are required when $\mathcal{E}$ is very small. This is difficult and sometimes impossible to handle such cases. Therefore, it is necessary to develop suitable numerical methods which are uniformly convergent to solve this type of differential equations. 
There are many papers in the literature dealing with the numerical approximation of the solution for singularly perturbed parabolic differential equations (SPPDEs) without delay (see [Miller et al., 1996; Miller et al., 1998; Clavero et al., 2000; Ansari et al., 2003; Aga et al., 2020) and the references therein) and with delay (see Patida and Sharma, 2006; Ansari et al., 2007; Bashier and Patidar, 2011a; Singh. et al., 2018; Bansal and Sharma, 2017 and 2018; Kumar et al., 2020; Mekonnen and File, 2021; Woldaregay and File, (Kragujevac Article in press), Daba and File, 2020 and the references therein). The existence and uniqueness of the second order parabolic delay differential equations with integral boundary conditions and its applications is discussed in Bahuguna and Dabas (2008). But, in recent years, uniformly convergent numerical methods for solving singularly perturbed delay ordinary differential equations with integral boundary conditions have been developed extensively in literature (see [Amiraliyev et al., (2017), Amiraliyev et.al., (2017), Habtamu.G. and File .G.(2020), Debela and Duressa.(2020), Sekar.E Tamilselvan.A (2019), Habtamu.G. and File .G.(2019), Amiraliyev GM and Ylmaz B.(2014), and Kudu, and Amiraliyev, (2015)] and the references therein). However, up to the best of our knowledge, except the work in [Elango et al., (2021)] not much work has been done to solve the problem under consideration. Hence, in this paper, motivated by the works of [Elango et al., (2021)], we construct and analyze a parameter uniform numerical method. The proposed method uses fitted operator finite difference method based on the procedures of method of line, which consists of non-standard finite difference operator for the spatial discretization and classical backward Euler method for the time discretization, which treat the problem and first being considered. The basic idea behind the method is to replace the denominator functions of the classical derivatives with positive functions derived in such a way that they capture some notable properties of the governing differential equation and hence provide the reliable numerical results (Patidar and Sharma, 2006).

The structure of the paper is as follows: In Section 2, the problem under study, some bounds for the analytical solution and its partial derivatives and the continuous maximum principles are given. In Section 3, discretizing the spatial domain and techniques of non-standard finite difference is discussed, and the $\varepsilon$-uniform convergence of the semi-discrete problem is proved. Next, classical backward Euler method used for the system of initial value problems resulted from spatial discretization and discuss the convergence of the discrete scheme. In Section 4, numerical results and discussion are given to validate the theoretical analysis. Finally, conclusion are included in Section 5, the conclusion of the work done is presented.

\section{Statement of the Problem}

Consider the following singularly perturbed delay parabolic second order differential equation with non- local boundary condition

$$
L u(x, t) \equiv-\varepsilon \frac{\partial^{2} u}{\partial x^{2}}+a(x, t) u(x, t)+b(x, t) u(x-1, t)+\frac{\partial u}{\partial t}=f(x, t), \quad \forall(x, t) \in \Omega
$$

Subject to initial condition

$$
u(x, t)=\phi_{b}(x, t), \quad(x, t) \in \Gamma_{b},
$$

and boundary conditions

$$
\left\{\begin{array}{c}
u(x, t)=\phi_{l}(x, t), \quad(x, t) \in \Gamma_{l}, \\
\kappa u(x, t)=u(2, t)-\varepsilon \int_{0}^{2} g(x) u(x, t) d x=\phi_{r}(x, t),(x, t) \in \Gamma_{r} .
\end{array}\right.
$$

On $\Omega=D \times(0, T]$, in space-time plane, where $D=(0,2), T$ is some fixed positive number and $\Gamma=\Gamma_{l} \cup \Gamma_{b} \cup \Gamma_{r}$. where $\Gamma_{l}=\{(x, t) ;-1 \leq x \leq 0$ and $0 \leq t \leq T\}$ and $\Gamma_{r}=\{(2,0) ; 0 \leq t \leq T\}$ are the left and the right sides of the rectangular domain $\Omega$ corresponding to $x=0$ and $x=2$, respectively, and the base of the domain is given by $\Gamma_{b}=\{(x, 0) ; 0 \leq x \leq 2\}$.

Where $\forall(x, t) \in \Omega, \bar{\Omega}=\bar{D} \times[0, T]$ and $\varepsilon \in(0,1)$ is given constant, $\quad a(x, t), b(x, t), f(x, t)$ on $\bar{\Omega}$ and $\phi_{l}(x, t)$, $\phi_{l}(x, t), \phi_{r}(x, t), \phi_{b}(x, t)$ on $\Gamma$ are sufficiently smooth, bounded functions that satisfy $a(x, t) \geq \alpha>0, b(x, t) \leq \beta<0$, $\alpha+\beta>0$, on $\bar{\Omega}$. Furthermore, $g(x)$ is non-negative function, monotonic and satisfy $1-\int_{0}^{2} g(x) d x>0$.

The problem (1) and (3) can be rewritten as,

$$
\operatorname{Lu}(x, t)=G(x, t)
$$




$$
\begin{gathered}
L u(x, t)= \begin{cases}-\varepsilon \frac{\partial^{2} u(x, t)}{\partial x^{2}}+a(x, t) u(x, t)+\frac{\partial u}{\partial t}, & \forall(x, t) \in \Omega_{1}, \\
-\varepsilon \frac{\partial^{2} u(x, t)}{\partial x^{2}}+a(x, t) u(x, t)+b(x, t) u(x-1, t)+\frac{\partial u}{\partial t}, & \forall(x, t) \in \Omega_{2} .\end{cases} \\
G(x, t)= \begin{cases}f(x, t)-b(x, t) \phi_{l}(x-1, t), & \forall(x, t) \in(0,1) \times(0, T], \\
f(x, t), & \forall(x, t) \in(1,2) \times(0, T] .\end{cases}
\end{gathered}
$$

with boundary conditions

$$
\left\{\begin{array}{l}
u(x, t)=\phi_{l}(x, t), \quad \forall(x, t) \in \Gamma_{l}, \\
u\left(1^{-}, t\right)=u\left(1^{+}, t\right), \quad u_{x}\left(1^{-}, t\right)=u_{x}\left(1^{+}, t\right), \\
u(x, t)=\phi_{b}(x, t), \quad \forall(x, t) \in \Gamma_{b}, \\
\kappa u(x, t)=u(2, t)-\varepsilon \int_{0}^{2} g(x) u(x, t) d x=\phi_{r}(x, t), \quad \forall(x, t) \in \Gamma_{r} .
\end{array}\right.
$$

where $\Omega_{1}=(0,1) \times[0, T], \Omega_{2}=(1,2) \times[0, T], \Omega^{*}=\Omega_{1} \cup \Omega_{2}$.

\section{Bounds on the Solution and its Derivative}

The existence and uniqueness of a solution for problem (5)-(7) can be established by assuming that the data are Holder continuous and imposing appropriate compatibility conditions at the corner points $(0,0),(2,0),(-1,0)$ and $(1,0)$ (see [Ladyzhenskaya O.A., et.al., (1968)]). Then, the required compatibility conditions are

$$
\phi_{b}(0,0)=\phi_{l}(0,0) \text { and } \phi_{b}(2,0)=\phi_{r}(2,0) .
$$

and

$$
\left\{\begin{array}{l}
-\left.\varepsilon \frac{\partial^{2} \phi_{b}}{\partial x^{2}}\right|_{(0,0)}+a(0,0) \phi_{b}(0,0)+b(0,0) \phi_{l}(-1,0)+\left.\frac{\partial \phi_{l}(0,0)}{\partial t}\right|_{(0,0)}=f(0,0), \\
-\left.\varepsilon \frac{\partial^{2} \phi_{b}}{\partial x^{2}}\right|_{(2,0)}+a(2,0) \phi_{b}(2,0)+b(2,0) \phi_{b}(1,0)+\left.\frac{\partial \phi_{r}(2,0)}{\partial t}\right|_{(2,0)}=f(2,0) .
\end{array}\right.
$$

so that the data matches at the corner points.

The following theorem gives sufficient conditions for the existence of a unique solution of the problem (5)-(7).

Theorem 3.1 Let $a, b, f \in C^{\left(\beta_{1}, \beta_{1} / 2\right)}(\bar{\Omega}), \phi_{l}, \phi_{r} \in C^{1+\beta_{1} / 2}([0, T]), \phi_{b} \in C^{\left(2+\beta_{1}, 1+\beta_{1} / 2\right)}\left(\Gamma_{b}\right), \beta_{1} \in(0,1)$. Then, the problem (5)-

(7) has a unique solution $u(x, t) \in C^{\left(2+\beta_{1}, 1+\beta_{1} / 2\right)}(\bar{\Omega})$. In particular, when the compatibility conditions (8) and (9) are not satisfied, a unique standard solution still exists but is not differentiable on all of $\partial \Omega$.

Proof: One may refer (see (Ladyzhenskaya et al., 1968)).

The reduced problem corresponding to singularly perturbed delay parabolic PDE (5)-(7) is given as:

$$
\begin{aligned}
& \left\{\begin{array}{l}
\left(u_{0}\right)_{t}+a(x, t) u_{0}(x, t)=f(x, t)-b(x, t) \phi_{l}(x-1, t), \quad \forall(x, t) \in \Omega_{1}, \\
u_{0}(x, t)=\phi_{b}(x, t), \quad \forall(x, t) \in \Gamma_{b} .
\end{array}\right. \\
& \left\{\begin{array}{l}
\left(u_{0}\right)_{t}+a(x, t) u_{0}(x, t)+b(x, t) u_{0}(x-1, t)=f(x, t), \quad \forall(x, t) \in \Omega_{2}, \\
u_{0}(x, t)=\phi_{b}(x, t), \quad \forall(x, t) \in \Gamma_{b} .
\end{array}\right.
\end{aligned}
$$

As $u_{0}(x, t)$ need not satisfy $u_{0}(0, t)=u(0, t)$ and $u_{0}(2, t)=u(2, t)$, the solution $u(x, t)$ exhibits boundary layers at $x=0$ and $x=2$. Further, as $u_{0}\left(1^{-}, t\right)$ need not be equal to $u_{0}\left(1^{+}, t\right)$, the solution $u(x, t)$ exhibits interior layers at $x=1$.

Lemma 3.1 The solution $u(x, t)$ of (5)-(7) satisfies the estimate 


$$
\left|u(x, t)-\phi_{b}(x, 0)\right| \leq C t, \forall(x, t) \in \bar{\Omega} .
$$

Where $C$ is a constant independent of $\mathcal{E}$.

Proof. The result follows from the compatibility condition. The detailed proof in [Roos.M. et.al, 2008]

Let $L$ be a differential operator that denotes the differential equation in (1)-(3) satisfies the following continuous maximum principle.

Lemma 3.2 (Continuous maximum Principle) Let $\Upsilon(x, t) \in U^{*}=C^{(0,0)}(\bar{\Omega}) \cap C^{(1,0)}(\Omega) \cap C^{(2,1)} \Omega^{*}$

such that $\Upsilon(0, t) \geq 0, \Upsilon(x, 0) \geq 0, \kappa \Upsilon(2, t) \geq 0, L_{1} \Upsilon(x, t) \geq 0, \forall(x, t) \in \Omega_{1}$,

$$
L_{2} \Upsilon(x, t) \geq 0, \forall(x, t) \in \Omega_{2} \text {, and }\left[\Upsilon_{x}\right](1, t)=\Upsilon_{x}\left(1^{+}, t\right)-\Upsilon_{x}\left(1^{-}, t\right) \leq 0 \text { then, } \Upsilon(x, t) \geq 0, \forall(x, t) \in \bar{\Omega} \text {. }
$$

Proof: For the proof one can refer [Elango.et.al, 2021]

Lemma 3.3 (Stability Result) The solution $u(x, t)$ of the problem (5)-(7), satisfies the bound

$$
\|u\|_{\bar{\Omega}} \leq C \max \left\{\|u\|_{\Gamma_{l}},\|u\|_{\Gamma_{b}},\|\kappa u\|_{\Gamma_{r}},\|L u\|_{\Omega^{*}}\right\},(x, t) \in \bar{\Omega} .
$$

Proof: For the proof one can refer [Elango.et.al, 2021]

Theorem 3.2 Let $a(x, t), b(x, t), f(x, t) \in C^{\left(2+\beta_{1}, 1+\beta_{1} / 2\right)}(\bar{\Omega}), \phi_{l} \in C^{\left(2+\beta_{1} / 2\right)}([0, T]), \phi_{r} \in C^{\left(2+\beta_{1} / 2\right)}([0, T])$ $\phi_{b} \in C^{\left(4+\beta_{1}, 2+\beta_{1} / 2\right)}\left(\Gamma_{b}\right), \beta_{1} \in(0,1)$. Assume that the compatibility conditions (8) and (9) are fulfilled. Then, the problem (5)-(7) has a unique solution $u(x, t)$ and $u \in C^{\left(4+\beta_{1}, 2+\beta_{1} / 2\right)}(\bar{\Omega})$. Furthermore, the derivatives of the solution $u$ satisfy:

$$
\left\|\frac{\partial^{(i+j)} u(x, t)}{\partial x^{i} \partial t^{j}}\right\|_{\bar{\Omega}} \leq C \varepsilon^{-i / 2}, \quad \forall i, j \in \square \geq 0 \text { Such that } 0 \leq i+2 j \leq 4,
$$

where the constant $C$ is independent of $\mathcal{E}$.

Proof: For the proof, refer to [Elango.et.al, 2021]

The following theorem provides the bound for the derivatives of the regular and the singular components respectively.

Theorem 3.3 Let the data $a, b, f \in C^{\left(4+\beta_{1}, 2+\beta_{1} / 2\right)}(\bar{\Omega}), \phi_{l}, \phi_{r} \in C^{3+\beta_{1} / 2}([0, T]), \phi_{b} \in C^{\left(6+\beta_{1}, 3+\beta_{1} / 2\right)}\left(\Gamma_{b}\right)$,

$\beta_{1} \in(0,1)$. Assume that the compatibility conditions (8) and (9) are satisfied. Then, we have

$$
\begin{gathered}
\left\|\frac{\partial^{(i+j)} v}{\partial x^{i} \partial t^{j}}\right\|_{\bar{\Omega}} \leq C\left(1+\varepsilon^{1-i / 2}\right), \\
\left\|\frac{\partial^{(i+j)} w_{l}(x, t)}{\partial x^{i} \partial t^{j}}\right\| \leq\left\{\begin{array}{l}
C \varepsilon^{-i / 2}(\exp (-x / \sqrt{\varepsilon})) \quad(x, t) \in \Omega_{1}, \\
C \varepsilon^{-i / 2}(\exp (-(x-1) / \sqrt{\varepsilon}))(x, t) \in \Omega_{2} .
\end{array}\right. \\
\left\|\frac{\partial^{(i+j)} w_{r}(x, t)}{\partial x^{i} \partial t^{j}}\right\| \leq\left\{\begin{array}{l}
C \varepsilon^{-i / 2}(\exp (-(1-x) / \sqrt{\varepsilon}))(x, t) \in \Omega_{1}, \\
C \varepsilon^{-i / 2}(\exp (-(2-x) / \sqrt{\varepsilon}))(x, t) \in \Omega_{2} .
\end{array}\right.
\end{gathered}
$$

where $C$ is constant independent of $\mathcal{E},(x, t) \in \bar{\Omega}, i, j \geq 0,0 \leq i+2 j \leq 4$.

Proof: One may refer [Elango.et.al, 2021] for the details.

\section{Formulation of the Numerical Scheme}

The theoretical basis of non-standard discrete modeling method is based on the development of exact finite difference method. In [28], Micken's presented techniques and rules for developing non-standard FDMs for different problem types. In Mickens's rules, to develop a discrete scheme, denominator function for the discrete derivatives must be expressed in terms of more complicated functions of step sizes than those used in the standard procedures. This complicated function constitutes a general property of the schemes, which is useful while designing reliable schemes for such problems. On the spatial domain $[0,2]$, we introduce the equidistant meshes with uniform mesh length $h$ such that 


$$
\Omega_{x}^{N}=\left\{0=x_{0}, x_{i}=i h, i=1(1) N-1, x_{N / 2}=1, x_{N}=2, h=\frac{2}{N}\right\} .
$$

where $h$ is the step size and $N$ is the number of mesh points in the spatial direction. Let we consider, the domain [0,1] which is discretized into $N / 2$ equal number of subintervals, each of length $h$. For problem (1)-(4), in order to construct exact finite difference scheme we follow the procedures used in [Mickens.R.E 2005].

Consider the constant coefficient sub equations given in (16) by ignoring the time variable for as

$$
-\varepsilon \frac{d^{2} u(x)}{d x^{2}}+\alpha u(x)=0
$$

where $a(x, t) \geq \alpha>0$. Thus the problem (16) has two independent solutions namely $\exp \left(\lambda_{1} x\right)$ and $\exp \left(\lambda_{2} x\right)$ with

$$
\lambda_{1,2}= \pm \sqrt{\alpha / \varepsilon} \text {. }
$$

we denote the approximate solution of $u(x)$ at $x_{i}^{\prime} s$ by $U_{i}$. Now our objective is to calculate a difference equation which has the same general solution as the problem (16) has at the grid point $x_{i}$ given by $U_{i}=A \exp \left(\lambda_{1} x_{i}\right)+B \exp \left(\lambda_{2} x_{i}\right)$. Using the procedures used in [Mickens.R.E 2005] we have

$$
\operatorname{det}\left(\begin{array}{ccc}
U_{i-1} & \exp \left(\lambda_{1} x_{i-1}\right) & \exp \left(\lambda_{2} x_{i-1}\right) \\
U_{i} & \exp \left(\lambda_{1} x_{i}\right) & \exp \left(\lambda_{2} x_{i}\right) \\
U_{i+1} & \exp \left(\lambda_{1} x_{i+1}\right) & \exp \left(\lambda_{2} x_{i+1}\right)
\end{array}\right)=0 .
$$

Simplifying the determinant in (18), we obtain that

$$
U_{i-1}-2 \cosh \left(\sqrt{\frac{\alpha}{\varepsilon}} h\right) U_{i}+U_{i+1}=0 .
$$

is an exact difference scheme for (16). After doing the arithmetic manipulation and rearrangement on (19) for the variable coefficient problem, we obtain

$$
-\varepsilon \frac{U_{i-1}-2 U_{i}+U_{i+1}}{\omega_{i}^{2}(\varepsilon, h, t)}+a_{i}(t) U_{i}=0
$$

where

$$
\omega_{i}=\frac{2}{\eta_{i}} \sinh \left(\frac{\eta_{i} h}{2}\right)
$$

with $\eta_{i}=\sqrt{\frac{a_{i}(t)}{\varepsilon}}$.

Consider (5)-(7) on the domain $\Omega=(0,2) \times[0, T]$, for $i=1, . ., N-1$

Let $U_{i}(t)$ denoted for the approximation of $u\left(x_{i}, t\right)$. By using the non-standard finite difference approximation (NSFD), at this stage the problem in (5)-(7) reduces to semi- discrete form as

$$
\begin{aligned}
& L^{N} U_{i}(t)=G_{i}(t), \text { for } i=1,2, \ldots, N-1 . \\
& \text { where } L^{N} U_{i}(t)= \begin{cases}-\mathcal{E} \frac{U_{i+1}(t)-2 U_{i}(t)+U_{i-1}(t)}{\omega_{i}^{2}(\varepsilon, h, t)}+a_{i}(t) U_{i}(t)+\frac{d U_{i}(t)}{d t}, & \forall\left(x_{i}, t\right) \in \Omega_{1}^{N} \times(0, T], \\
-\mathcal{E} \frac{U_{i+1}(t)-2 U_{i}(t)+U_{i-1}(t)}{\omega_{i}^{2}(\varepsilon, h, t)}+a_{i}(t) U_{i}(t)+b_{i}(t) U\left(x_{i-N / 2}, t\right)+\frac{d U_{i}(t)}{d t}, & \forall\left(x_{i}, t\right) \in \Omega_{2}^{N} \times(0, T] .\end{cases} \\
& G_{i}(t)= \begin{cases}f_{i}(t)-b_{i}(t) \phi_{l}\left(x_{i-N / 2}, t\right), & \forall\left(x_{i}, t\right) \in \Omega_{1}^{N} \times(0, T], \\
f_{i}(t), & \forall\left(x_{i}, t\right) \in \Omega_{2}^{N} \times(0, T] .\end{cases}
\end{aligned}
$$


For $i=N$, the composite Simpson's integration rule approximates the non-local boundary condition in (3) as follows:

$$
u(2, t)-\varepsilon \int_{0}^{2} g(x) u(x, t) d x=U_{N}(t)-\varepsilon h \sum_{i=0}^{N} c_{i} g_{i} U_{i}(t)=\phi_{r}, \quad 0<t \leq T,
$$

where $c_{0}=c_{N}=\frac{1}{3}, c_{2 k-1}=\frac{4}{3}$, for $k=1,2, . ., \frac{N}{2}$ and $c_{2 k}=\frac{2}{3}$, for $k=1,2, . ., \frac{N}{2}-1$,

Now, using (25) and left boundary condition, we can obtain the following formula for $U_{N}(t)$

$$
\begin{aligned}
U_{N}(t) & =\frac{1}{\left(1-\varepsilon h c_{N} g_{N}\right)}\left[\varepsilon h \sum_{i=0}^{N-1} c_{i} g_{i} U_{i}(t)+\phi_{r}\right], \\
& =\frac{1}{\left(1-\varepsilon h c_{N} g_{N}\right)}\left[\varepsilon h c_{0} g_{0} \phi_{l}(0, t)+\varepsilon h \sum_{i=1}^{N-1} c_{i} g_{i} U_{i}(t)+\phi_{r}\right] .
\end{aligned}
$$

At this stage the time domain is continuous and the system of IVPs in (23), (24) and (26) can be written in compact form as

$$
\frac{d U_{i}(t)}{d t}+H(t) U_{i}(t)=G_{i}(t)
$$

where $H(t)$ is a matrix of size $N x N$ and $U_{i}(t)$ and $\mathrm{G}_{i}(t)$ are vectors of size $N$. The entries of the coefficient matrix $H(t)$ are respectively given by

$$
\begin{cases}H_{i i-1}(t)=\frac{-\varepsilon}{\omega_{i}^{2}(\varepsilon, h, t)}, & \text { for } i=2,3, \ldots, N-1, \\ H_{i i}(t)=\frac{2 \varepsilon}{\omega_{i}^{2}(\varepsilon, h, t)}+a_{i}(t), & \text { for } i=1,2, \ldots, N-1, \\ H_{i i+1}(t)=\frac{-\varepsilon}{\omega_{i}^{2}(\varepsilon, h, t)}, & \text { for } i=1,2, . ., N-1, \\ H_{i i-N / 2}(t)=b_{i}(\mathrm{t}), & \text { for } i=N / 2+1, \ldots, N-1 .\end{cases}
$$

and the corresponding right-hand side vector in the equation system has the entries

$$
\left\{\begin{array}{l}
G_{1}(t)=f_{1}(t)-b_{1}(t) \phi_{l}\left(x_{1-N / 2}, t\right)+\left(\frac{\varepsilon}{\omega_{1}^{2}(\varepsilon, h, t)}\right) \phi_{l}(0, t), \\
G_{i}(t)= \begin{cases}f_{i}(t)-b_{i}(t) \phi_{l}\left(x_{i-N / 2}, t\right), & \text { for } i=2,3, . ., N / 2, \\
f_{i}(t), & \text { for } i=N / 2+1, \ldots, N-1 .\end{cases}
\end{array}\right.
$$

Before we proceed with the convergence analysis, we highlight some properties of the discrete problem to the original (1)-(3) in the form of lemma which play a major role in the said analysis.

For $i=1,2, \ldots, N / 2$,

$$
L_{1}^{N} U_{i}(t)=f_{i}(t)-b_{i}(t) \phi_{l}\left(x_{i-N / 2}, t\right) .
$$

For $i=N / 2+1, \ldots, N-1$,

$$
L_{2}^{N} U_{i}(t)=f_{i}(t)
$$

Subject to the boundary conditions:

$$
\left\{\begin{array}{l}
U_{i}(t)=\phi_{l}\left(x_{i}, t\right), \quad i=-N / 2-N / 2+1, \ldots, 0 \text { and } t \in[0, T] \\
D_{x}^{-} U\left(x_{N / 2}, t\right)=D_{x}^{+} U\left(x_{N / 2}, t\right) \\
U_{i}(t)=\phi_{b}\left(x_{i}, t\right), \quad i=1,2, \ldots, N-1 .
\end{array}\right.
$$

For $i=N, \kappa^{N} U\left(x_{N}, t\right)=U\left(x_{N}, t\right)-\varepsilon \sum_{i=1}^{N} \frac{g_{i-1} U_{i-1}(t)+4 g_{i} U_{i}(t)+g_{i+1} U_{i+1}(t)}{3} h_{i}$. 
where, $L_{1}^{N} U_{i}(t)=U_{i}^{\prime}(t)-\varepsilon \delta^{2} U_{i}(t)+a_{i}(t) U_{i}(t)$.

$$
L_{2}^{N} U_{i}(t)=U_{i}^{\prime}(t)-\varepsilon \delta^{2} U_{i}(t)+a_{i}(t) U_{i}(t)+b_{i}(t) U_{i-N / 2}(t) .
$$

\section{Convergence Analysis}

The problem (23), (24) and (26), satisfies the following well-known semi-discrete maximum principle on $\bar{\Omega}_{\tau}^{N} \times[0, T]$.

Lemma 5.1 (Semi-discrete maximum principle) Assume that

$$
\sum_{i=1}^{N} \frac{g_{i-1}+4 g_{i}+g_{i+1}}{3} h_{i}=\rho<1
$$

and mesh function $\Theta_{i}(t)$ satisfies $\Theta_{0}(t) \geq 0, \Theta_{i}(t) \geq 0$ and $\kappa^{N} \Theta_{N}(t) \geq 0$, Then $L_{1}^{N} \Theta_{i}(t) \geq 0$,

$\forall\left(x_{i}, t\right) \in \Omega_{1}^{N} \times[0, T], L_{2}^{N} \Theta_{i}(t) \geq 0, \forall\left(x_{i}, t\right) \in \Omega_{2}^{N} \times[0, T]$, and $\left[D_{x}\right] \Theta\left(x_{N / 2}, t\right)=D_{x}^{+} \Theta\left(x_{N / 2}, t\right)-D_{x}^{-} \Theta\left(x_{N / 2}, t\right) \leq 0$ imply that $\Theta_{i}(t) \geq 0, \forall\left(x_{i}, t\right) \in \bar{\Omega}^{N} \times[0, T]$.

Proof: Define a test function $S\left(x_{i}, t\right)$ as

$$
S\left(x_{i}, t\right)=\left\{\begin{array}{l}
\frac{1}{8}+\frac{x_{i}}{2},\left(x_{i}, t\right) \in \Omega_{1}^{N} \times[0, T] \\
\frac{3}{8}+\frac{x_{i}}{4},\left(x_{i}, t\right) \in \Omega_{2}^{N} \times[0, T]
\end{array}\right.
$$

Note that

$S\left(x_{i}, t\right)>0, \forall\left(x_{i}, t\right) \in \bar{\Omega}^{N} \times[0, T], L^{N} S\left(x_{i}, t\right)>0, \forall\left(x_{i}, t\right) \in\left(\Omega_{1}^{N} \cup \Omega_{2}^{N}\right) \times[0, T], S\left(x_{0}, t\right)>0, S\left(x_{i}, t\right)>0$, $k^{N} S\left(x_{N}, t\right)>0$, and $\left[D_{x}\right] S\left(x_{N / 2}, t\right)<0$. Let

$$
\gamma=\max \left\{\frac{-x\left(x_{i}, t\right)}{S\left(x_{i}, t\right)}:\left(x_{i}, t\right) \in \bar{\Omega}^{N} \times[0, T]\right\} .
$$

Then, there exists $\left(x^{*}, t\right) \in \bar{\Omega}^{N} \times[0, T]$ such that $\Theta\left(x^{*}, t\right)+\gamma S\left(x^{*}, t\right)=0$ and $\Theta\left(x_{i}, t\right)+\gamma S\left(x_{i}, t\right) \geq 0$,

$\forall\left(x_{i}, t\right) \in \bar{\Omega}^{N} \times[0, T]$. Therefore, the function attains its minimum at $(x, t)=\left(x^{*}, t\right)$. suppose the theorem does not hold true, then $\gamma>0$,

Case (i): $\left(x^{*}, t\right)=\left(x_{0}, t\right), 0<(x+\gamma S)\left(x_{0}, t\right)=\Theta\left(x_{0}, t\right)+\gamma S\left(x_{0}, t\right)=0$, It is a contradiction.

Case (ii): $\left(x^{*}, t\right) \in \Omega_{1}^{N} \times[0, T], 0<L_{1}^{N}(x+\gamma S)\left(x^{*}, t\right)=\left(-\varepsilon \delta_{x}^{2} D_{t}+a\right)(x+\gamma S)\left(x^{*}, t\right) \leq 0$, It is a contradiction.

Case (iii): $\left(x^{*}, t\right)=\left(x_{N / 2}, t\right), 0 \leq\left[D_{x}(x+\gamma S)\right]\left(x_{N / 2}, t\right)<0$, It is a contradiction.

Case (iv): $\left(x^{*}, t\right) \in \Omega_{2}^{N} \times[0, T] 0<L_{2}^{N}(x+\gamma S)\left(x^{*}, t\right)=\left(-\varepsilon \delta_{x}^{2}+D_{t}+a\right)(x+\gamma S)\left(x^{*}, t\right)+b(x+\gamma S)\left(x^{*}-x_{N / 2}, t\right) \leq 0$

It is a contradiction.

Case (v): $\left(x^{*}, t\right)=\left(x_{N}, t\right)$

$$
0<k^{N}(x+\gamma S)\left(x_{N}, t\right)=(x+\gamma S)\left(x_{N}, t\right)-\varepsilon \sum_{i=1}^{N} \frac{g_{i-1}(x+\gamma S)_{i-1}(t)+4 g_{i}(x+\gamma S)_{i}(t)+g_{i+1}(x+\gamma S)_{i+1}(t)}{3} h_{i} \leq 0 .
$$

It is a contradiction.

Hence, the proof of the theorem

Lemma 5.2 Let $\Theta_{i}(t)$ be any mesh function then,

$$
\left\|\Theta_{i}(t)\right\|_{\bar{\Omega}^{N} \times[0, T]} \leq C \max \left\{\left\|\Theta_{i}(t)\right\|_{\Gamma_{l}^{N} \times[0, T]},\left\|\Theta_{i}(t)\right\|_{\Gamma_{b}{ }^{N} \times[0, T]},\left\|\kappa^{N} \Theta_{i}(t)\right\|_{\Gamma_{r}{ }^{N} \times[0, T]}, \max _{\left(x_{i}, t\right) \in\left(\Omega_{1}^{N} \cup \Omega_{2}^{N}\right) \times[0, T]}\left\|L^{N} \Theta_{i}(t)\right\|\right\},
$$

Proof: Consider the barrier functions 


$$
\vartheta^{ \pm}\left(x_{i}, t\right)=C M S\left(x_{i}, t\right) \pm \Theta\left(x_{i}, t\right),\left(x_{i}, t\right) \in \bar{\Omega}^{N} \times[0, T],
$$

where

$$
M=\max \left\{\|\Theta\|_{\Gamma_{l}^{N} \times[0, T]},\|\Theta\|_{\Gamma_{b}{ }^{N} \times[0, T]},\left\|\kappa^{N} \Theta\right\|_{\Gamma_{r}^{N} \times[0, T]}, \max _{\left(x_{i}, t\right) \in\left(\Omega_{1}^{N} \cup \Omega_{2}^{N}\right) \times[0, T]}\left\|L^{N} \Theta\right\|\right\}
$$

and $S\left(x_{i}, t\right)$ is the test function as in Lemma 5.1.

From $(31)$ it is clear that $\vartheta^{ \pm}\left(x_{0}, t\right) \geq 0, \vartheta^{ \pm}\left(x_{i}, t\right) \geq 0$ and $\kappa^{N} \vartheta^{ \pm}\left(x_{N}, t\right) \geq 0 . L_{1}^{N} \vartheta^{ \pm}\left(x_{i}, t\right) \geq 0, \forall\left(x_{i}, t\right) \in \Omega_{1}^{N}$, $L_{2}^{N} \vartheta^{ \pm}\left(x_{i}, t\right) \geq 0, \forall\left(x_{i}, t\right) \in \Omega_{2}^{N}, D_{x}^{+} \vartheta^{ \pm}\left(x_{N / 2}, t\right)-D_{x}^{-} \vartheta^{ \pm}\left(x_{N / 2}, t\right) \leq 0$. using lemma5.2, $\vartheta^{ \pm}\left(x_{i}, t\right) \geq 0$, $\forall\left(x_{i}, t\right) \in \bar{\Omega}^{N} \times[0, T]$.

Now, let us analyze the error estimate of the spatial discretization. We proved above the discrete problem satisfy the maximum principle and the uniform stability estimate. Note that $U_{i}(t)$ is denoted for the spatial discretization approximate solution to the exact solution $u(x, t)$ at $x=x_{i}, i=0,1, \ldots, N$

Lemma 5.3 For a fixed mesh and $\mathcal{E} \rightarrow 0$, it holds:

$$
\lim _{\varepsilon \rightarrow 0} \max _{1<i<N / 2} \frac{\exp \left(-C x_{i} / \sqrt{\varepsilon}\right)}{\varepsilon^{m / 2}}=0, \quad m=1,2, \ldots
$$

and

$$
\lim _{\varepsilon \rightarrow 0} \max _{1<i<N / 2} \frac{\exp \left(-C\left(1-x_{i}\right) / \sqrt{\varepsilon}\right)}{\varepsilon^{m / 2}}=0, \quad m=1,2, \ldots,
$$

where $x_{i}=i h \quad h=2 / N$ for all $i=1,2, \ldots, N / 2$.

Proof: One may refer [Woldaregay.M and File.G. Kragujevac Article in press] for the details

Now, the truncation error of the scheme (23), (24) and (26) for $\left(x_{i}, t\right) \in \Omega_{1}^{N} \times[0, T]$ is given by

$$
\begin{aligned}
L_{1}^{N}\left(U_{i}(t)-u_{i}(t)\right) & =f_{i}(t)-b_{i}(t) \phi_{l}\left(x_{i-N / 2}, t\right)-L_{1}^{N} u_{i}(t), \\
& =\left(L_{1}-L_{1}^{N}\right) u_{i}(t), i=1(1) \frac{N}{2}, \\
& =-\varepsilon\left(u_{x x}(t)\right)_{i}+\frac{\varepsilon}{\omega_{i}^{2}(\varepsilon, h, t)}\left(u_{i+1}(t)-2 u_{i}(t)+u_{i-1}(t)\right), \quad i=1(1) \frac{N}{2} .
\end{aligned}
$$

Using the truncated Taylor series expansions of the terms $u_{i+1}(t)$ and $u_{i-1}(t)$ yields

$$
L_{1}^{N}\left(U_{i}(t)-u_{i}(t)\right)=-\varepsilon u_{x x}(t)+\frac{\varepsilon}{\omega_{i}^{2}(\varepsilon, h, t)}\left(h^{2}\left(u_{x x}(t)\right)_{i}+\frac{h^{4}}{12}\left(u_{x x x x}(t)\right)_{i} \xi_{i}\right) \xi_{i} \in\left(u_{i-1}, u_{i+1}\right) .
$$

Next we use a truncated Taylor series expansion of the denominator function, $1 / \omega_{i}^{2}$ of order five gives

$$
\frac{1}{\omega_{i}^{2}}=\frac{1}{h^{2}}-\frac{\rho_{i}^{2}}{12}+\frac{\rho_{i}^{4} h^{2}}{240}
$$

Now, substituting (33) in to (32), we obtain

$$
\begin{aligned}
L_{1}^{N}\left(U_{i}(t)-u_{i}(t)\right) & =\left(\frac{\varepsilon}{12}\left(\left(u_{x x x x}(t)\right)_{i} \xi_{i}-\rho_{i}^{2}\left(u_{x x}(t)\right)\right)_{i}\right) h^{2}+\frac{\varepsilon \rho_{i}^{2}}{48}\left(\frac{\rho_{i}^{2}}{5}\left(u_{x x}(t)\right)_{i}-\frac{1}{3}\left(u_{x x x x}(t)\right)_{i} \xi_{i}\right) h^{4} \\
& +\left(\varepsilon \frac{\rho_{i}^{4}}{2880}\left(u_{x x x x}(t)\right)_{i} \xi_{i}\right)_{i} h^{6}
\end{aligned}
$$

Using the bounds on the derivatives and Lemma 5.3 gives 


$$
L_{1}^{N}\left(U_{i}(t)-u_{i}(t)\right) \leq \frac{\varepsilon}{12}\left(1-\rho_{i}^{2}\right) h^{2}-\frac{\varepsilon \rho_{i}^{2}}{48}\left(\frac{1}{3}-\frac{\rho_{i}^{2}}{5}\right) h^{4}+\left(\frac{\varepsilon \rho_{i}^{4}}{2880}\right) h^{6} \leq C h^{2} .
$$

where we have used the relation $h^{2}>h^{4}>h^{6} \ldots$ with lemma (5.2) gives

$$
\left\|L_{1}^{N}\left(U_{i}(t)-u_{i}(t)\right)\right\| \leq C h^{2} .
$$

Hence, by discrete maximum principle, we obtain:

$$
\left\|U_{i}(t)-u_{i}(t)\right\| \leq C h^{2} .
$$

Remark: Similar analysis may be carried out for the finite difference scheme for $\left(x_{i}, t\right) \in \Omega_{2}^{N} \times[0, T]$, and for the right boundary condition.

\section{Discretization in Temporal Direction}

We discretized the IVPs (23), (24) and (25) with the classical Backward Euler method on a uniform mesh. Now denote the approximation of $u_{i}\left(t_{j}\right):=u_{i}^{j}$ by $U_{i}^{j}$. We perform the time discretization as follows:

Let $M$ be a positive integer and $t_{0}=0, t_{j}=t_{0}+j \Delta t, \Delta t=t_{j}-t_{j-1}, j=1(1) M-1, t_{M}=T$, where $M$ denotes the number of mesh points in time direction, then

$$
\frac{U^{j}-U^{j-1}}{\Delta t}+H\left(t_{j}\right) U^{j}=G\left(t_{j}\right), \text { for } j=1,2, \ldots, M,
$$

with initial condition $U^{(0)}=\phi_{l}\left(t_{j}\right)$. and rearranging equation (35) gives

$$
U^{j}=\left(I+\Delta t H\left(t_{j}\right)\right)^{-1}\left(\Delta t G\left(t_{j}\right)+U^{j-1}\right) .
$$

Lemma 6.1 The local truncation error associated with the time integration satisfies

$$
\left\|e_{j}\right\| \leq C(\Delta t)^{2}
$$

where $C$ is a constant independent of the perturbation parameter $\varepsilon$ and $M$.

Proof: The local truncation error is defined as

$$
\begin{aligned}
e_{j} & =u\left(t_{j}\right)-U^{j} \\
& =u\left(t_{j}\right)-\left[I+\Delta t H\left(t_{j}\right)\right]^{-1}\left(\Delta t G\left(t_{j}\right)+u\left(t_{j-1}\right)\right) .
\end{aligned}
$$

a Taylor series expansions of $u\left(t_{j-1}\right)$ takes the form

$$
u\left(t_{j-1}\right)=u\left(t_{j}\right)-\Delta t u_{t}\left(t_{j}\right)+\frac{(\Delta t)^{2}}{2} u_{t t}\left(t_{j}\right)-\frac{(\Delta t)^{3}}{6} u_{t t t}\left(t_{j}\right)+O(\Delta t)^{4}
$$

and

$$
\begin{aligned}
& u_{t}\left(t_{j}\right)=G\left(t_{j}\right)-H\left(t_{j}\right) u\left(t_{j}\right), \\
& u\left(t_{j-1}\right)=u\left(t_{j}\right)-(\Delta t)\left(G\left(t_{j}\right)-H\left(t_{j}\right) u\left(t_{j}\right)\right)+\frac{(\Delta t)^{2}}{2} u_{t t}\left(t_{j}\right)-\frac{(\Delta t)^{3}}{6} u_{t t t}\left(t_{j}\right)+O(\Delta t)^{4}
\end{aligned}
$$

The local truncation error Subtracting $e_{j}$ yields

$$
\begin{aligned}
e_{j} & =u\left(t_{j}\right)-\left[I+(\Delta t) H\left(t_{j}\right)\right]^{-1}\left(\left(I+(\Delta t) H\left(t_{j}\right)\right) u\left(t_{j}\right)+\frac{(\Delta t)^{2}}{2} u_{t t}\left(t_{j}\right)-\frac{(\Delta t)^{3}}{6} u_{t t t}\left(t_{j}\right)+O(\Delta t)^{4}\right) \\
& =\left[I+(\Delta t) H\left(t_{j}\right)\right]^{-1}\left(\frac{(\Delta t)^{2}}{2} u_{t t}\left(t_{j}\right)-\frac{(\Delta t)^{3}}{6} u_{t t t}\left(t_{j}\right)+O(\Delta t)^{4}\right)
\end{aligned}
$$

Since the matrix $H(t)$ is invertible, using the relation $(\Delta t)^{2}>(\Delta t)^{3}$ for small $(\Delta t)$ and $u\left(t_{j}\right) \leq C$, we obtain 


$$
\left\|e_{j}\right\|=\left\|\left(I+(\Delta t) H\left(t_{j}\right)\right)^{-1}\right\|(\Delta t)^{2} \leq C(\Delta t)^{2}
$$

which completes the proof

Lemma 6.2 The global error estimates in this temporal direction is given by

$$
\left\|T E_{j}\right\|=\max _{j}\left\|U_{i}\left(t_{j}\right)-U_{i}^{j}\right\|_{\Omega_{x}^{N} \times \Lambda_{t}^{M}} \leq C(\Delta t),
$$

where $\left\|T E_{j}\right\|$ is the global error in the temporal direction at $(j)^{\text {th }}$ time level.

Proof: One may refer [Woldaregay. M and File.G Kragujevac Article in press] for the details.

Since $C$ and $(\Delta t)$ are independent of the perturbation parameter $\mathcal{E}$, taking the suprimum for all $\mathcal{E} \in(0,1]$ we obtain.

$$
\sup _{0<\varepsilon \leq 1} \max _{j}\left\|U_{i}\left(t_{j}\right)-U_{i}^{j}\right\|_{\Omega_{x}^{N} \times \Lambda_{t}^{M}} \leq C(\Delta t)
$$

This shows that the discretization in time direction is consistent and global error is bounded, with the error bound $\Delta t$. Now, we use (34) and (40) to prove the parameter-uniform convergence of the fully discrete scheme as

$$
\begin{aligned}
\sup _{0<\varepsilon \leq 1} \max _{i, j}\left\|u\left(x_{i}, t_{j}\right)-U_{i}^{j}\right\| & =\sup _{0<\varepsilon \leq 1} \max _{i, j}\left\|u\left(x_{i}, t_{j}\right)-U_{i}\left(t_{j}\right)+U_{i}\left(t_{j}\right)-U_{i}^{j}\right\|, \\
& \leq \sup _{0<\varepsilon \leq 1} \max _{i, j}\left\|u\left(x_{i}, t_{j}\right)-U_{i}\left(t_{j}\right)\right\|+\sup _{0<\varepsilon \leq 1} \max _{i, j}\left\|U_{i}\left(t_{j}\right)-U_{i}^{j}\right\| .
\end{aligned}
$$

Hence, we obtain the required bound as follows

$$
\sup _{0<\varepsilon \leq 1}\left\|u\left(x_{i}, t_{j}\right)-U_{i}^{j}\right\|_{\Omega_{x}^{N} \times \Lambda_{t}^{M}} \leq C\left(h^{2}+(\Delta t)\right)
$$

Thus, the inequality in (41) shows the parameter uniform convergence of the proposed scheme with order of convergence: second order in spatial direction and first order in temporal direction.

\section{Numerical Examples and Results}

To validate the established theoretical results, we perform numerical experiments using the proposed numerical scheme on the problem given in (1)-(3). We consider two numerical examples to verify the parameter uniform convergence of the proposed scheme. Exact solution is not available for these two problems, therefore maximum nodal errors are calculated by using the double mesh technique as

$$
E_{\varepsilon}^{N, \Delta t}=\max _{i, j}\left|U_{i, j}^{N, \Delta t}-U_{i, j}^{2 N, \Delta t / 2}\right|
$$

For any values of mesh points $\mathrm{N}$ and $\Delta t$, the parameter uniform error estimate are calculated by

$$
E^{N, \Delta t}=\max _{\varepsilon}\left|E_{\varepsilon}^{N, \Delta t}\right|
$$

The rate of convergence of the scheme is calculated by the formula

$$
r_{\varepsilon}^{N, \Delta t}=\log _{2}\left(E_{\varepsilon}^{N, \Delta t} / E_{\varepsilon}^{2 N, \Delta t / 2}\right)=\frac{\log \left(E_{\varepsilon}^{N, \Delta t}\right)-\log \left(E_{\varepsilon}^{2 N, \Delta t / 2}\right)}{\log _{2}}
$$

and the parameter-uniform rate of convergence is calculated by:

$$
r^{N, \Delta t}=\log _{2}\left(E^{N, \Delta t} / E^{2 N, \Delta t / 2}\right)=\frac{\log \left(E^{N, \Delta t}\right)-\log \left(E^{2 N, \Delta t / 2}\right)}{\log _{2}}
$$

The numerical results are presented for the value of the perturbation parameter $\varepsilon \in\left\{10^{-6}, 10^{-7}, \ldots, 10^{-10}\right\}$

Example 1: Consider the following singularly perturbed problem [Elango.et.al, 2021]

$$
-\varepsilon \frac{\partial^{2} u}{\partial x^{2}}+5 u(x, t)-u(x-1, t)+\frac{\partial u}{\partial t}=e^{-x}, \quad(x, t) \in(0,2) \times(0,2],
$$

Subject to initial and boundary condition 


$$
\left\{\begin{array}{c}
u(x, t)=0, \quad \forall(x, t) \in \Gamma_{l}, \\
\kappa u(2, t)=u(2, t)-\varepsilon \int_{0}^{2} \frac{x}{3} u(x, t) d x=0, \quad \forall(x, t) \in \Gamma_{r}, \\
u(x, t)=0, \quad \forall(x, t) \in \Gamma_{b},
\end{array}\right.
$$

Example 2: Consider the following singularly perturbed problem

$$
-\varepsilon \frac{\partial^{2} u}{\partial x^{2}}+5 u(x, t)-x u(x-1, t)+\frac{\partial u}{\partial t}=1, \quad(x, t) \in(0,2) \times(0,2],
$$

Subject to initial and boundary condition

$$
\left\{\begin{array}{c}
u(x, t)=0, \quad \forall(x, t) \in \Gamma_{l}, \\
\kappa u(2, t)=u(2, t)-\varepsilon \int_{0}^{2} \frac{1}{6} u(x, t) d x=0, \quad \forall(x, t) \in \Gamma_{r}, \\
u(x, t)=\sin (\pi x), \quad \forall(x, t) \in \Gamma_{b} .
\end{array}\right.
$$

Table 1: Maximum absolute errors and rate of convergence for Example 1 at number of mesh points $N$ and $\Delta t$

\begin{tabular}{cccccc}
\hline$\varepsilon \downarrow$ & $N=16$ & $N=32$ & $N=64$ & $N=128$ & $N=256$ \\
& $\Delta t=0.1 / 2$ & $\Delta t=0.1 / 2^{2}$ & $\Delta t=0.1 / 2^{3}$ & $\Delta t=0.1 / 2^{4}$ & $\Delta t=0.1 / 2^{5}$ \\
\hline & & & & & \\
$10^{-6}$ & $3.5045 \mathrm{e}-03$ & $2.0026 \mathrm{e}-03$ & $1.0723 \mathrm{e}-03$ & $5.5505 \mathrm{e}-04$ & $2.8241 \mathrm{e}-04$ \\
$10^{-7}$ & $3.5045 \mathrm{e}-03$ & $2.0026 \mathrm{e}-03$ & $1.0723 \mathrm{e}-03$ & $5.5505 \mathrm{e}-04$ & $2.8241 \mathrm{e}-04$ \\
$10^{-8}$ & $3.5045 \mathrm{e}-03$ & $2.0026 \mathrm{e}-03$ & $1.0723 \mathrm{e}-03$ & $5.5505 \mathrm{e}-04$ & $2.8241 \mathrm{e}-04$ \\
$10^{-9}$ & $3.5045 \mathrm{e}-03$ & $2.0026 \mathrm{e}-03$ & $1.0723 \mathrm{e}-03$ & $5.5505 \mathrm{e}-04$ & $2.8241 \mathrm{e}-04$ \\
$10^{-10}$ & $3.5045 \mathrm{e}-03$ & $2.0026 \mathrm{e}-03$ & $1.0723 \mathrm{e}-03$ & $5.5505 \mathrm{e}-04$ & $2.8241 \mathrm{e}-04$ \\
\hline
\end{tabular}

Table 2: Comparisons of maximum absolute errors and rate of convergence for Example 1 at number of mesh

\begin{tabular}{cccccc}
\multicolumn{5}{c}{ points $\mathrm{N}$ and $\Delta t$ with $\mathrm{T}=2$, for $\varepsilon \in\left\{10^{-10}, 10^{-9}, \ldots, 10^{-6}\right\}}$. \\
\hline$N=16$ & $N=32$ & $N=64$ & $N=128$ & $N=256$ \\
$\Delta t=0.1 / 2$ & $\Delta t=0.1 / 2^{2}$ & $\Delta t=0.1 / 2^{3}$ & $\Delta t=0.1 / 2^{4}$ & $\Delta t=0.1 / 2^{5}$ \\
\hline $\begin{array}{c}\text { resent Method } \\
E^{N, \tau}\end{array}$ & $3.5045 \mathrm{e}-03$ & $2.0026 \mathrm{e}-03$ & $1.0723 \mathrm{e}-03$ & $5.5505 \mathrm{e}-04$ & $2.8241 \mathrm{e}-04$ \\
$r^{N, \tau}$ & 0.80733 & 0.90117 & 0.95002 & 0.97483 & 0.98734 \\
Results in Elango et al., $(2021)$ & & & & \\
$E^{N, \Delta t}$ & $2.0615 \mathrm{e}-02$ & $1.2534 \mathrm{e}-02$ & $6.9738 \mathrm{e}-03$ & $3.6873 \mathrm{e}-03$ & $1.8972 \mathrm{e}-03$ \\
$r^{N, \Delta t}$ & 0.71783 & 0.84584 & 0.91937 & 0.95873 & 0.97912 \\
\hline
\end{tabular}




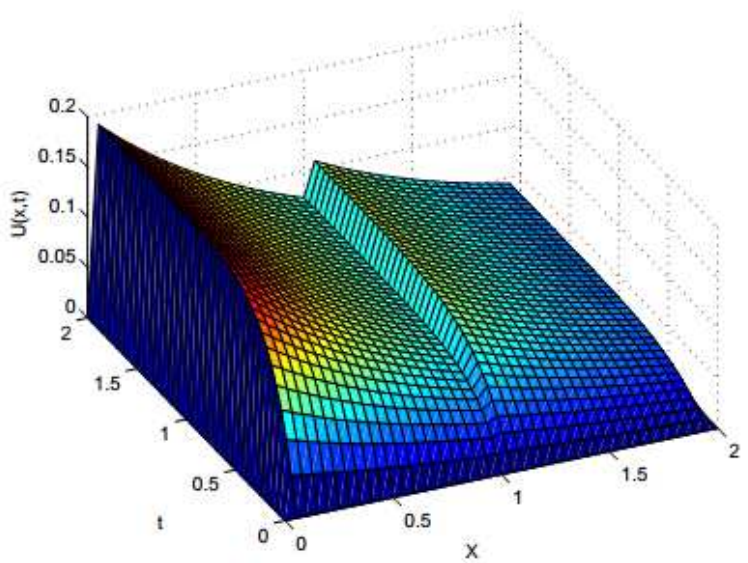

(a)

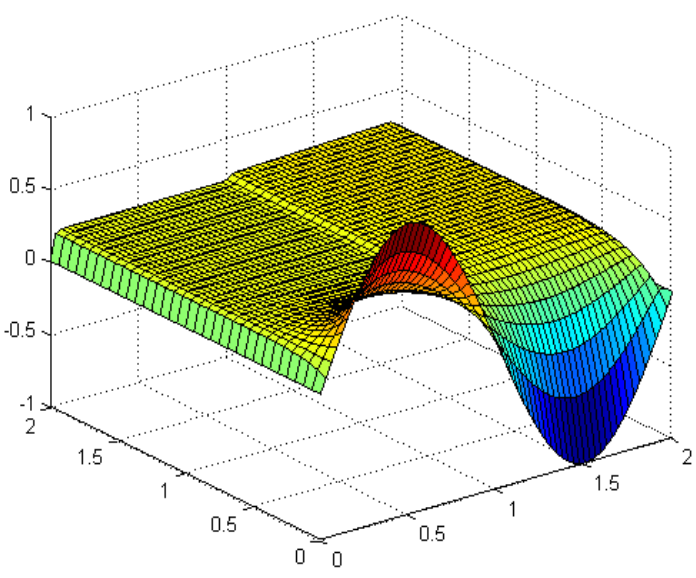

(b)

Figure. 1 Surface plot of the numerical solution for Example 1 on the left side and Example 2 in the right side for $N=M=32$ with boundary layer formation when $\varepsilon=10^{-3}$

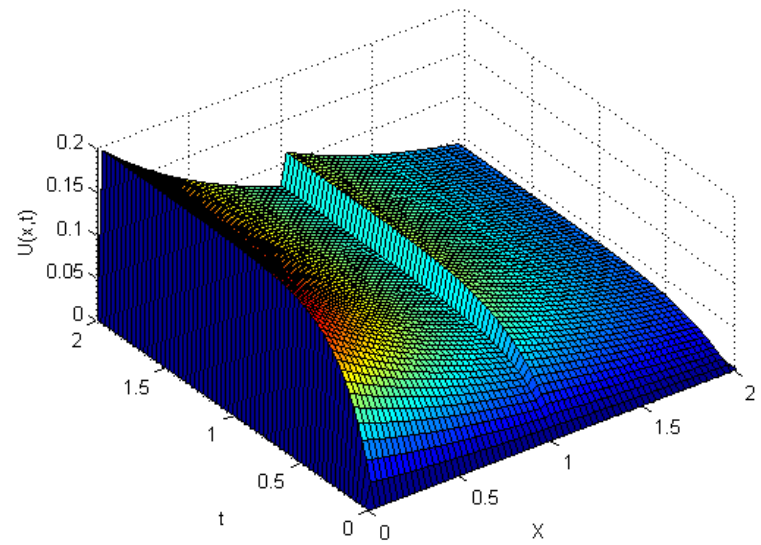

(a)

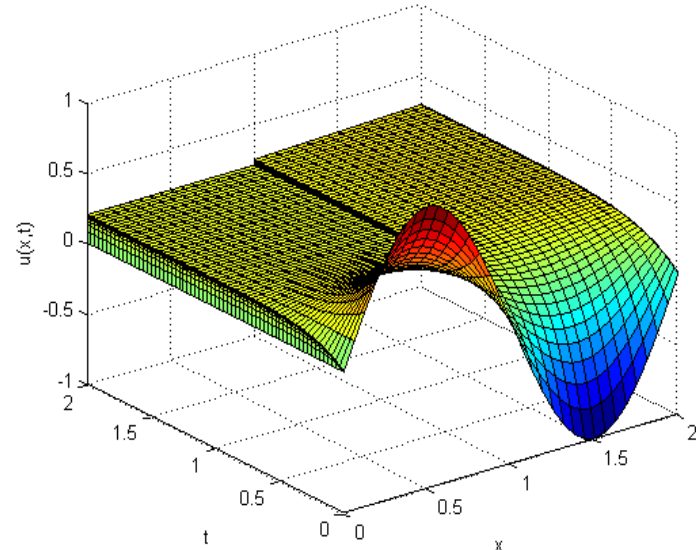

(b)

Figure. 2 Surface plot of the numerical solution for Example 1 on the left side and Example 2 in the right side for $N=M=64$ with boundary layer formation when $\varepsilon=10^{-10}$

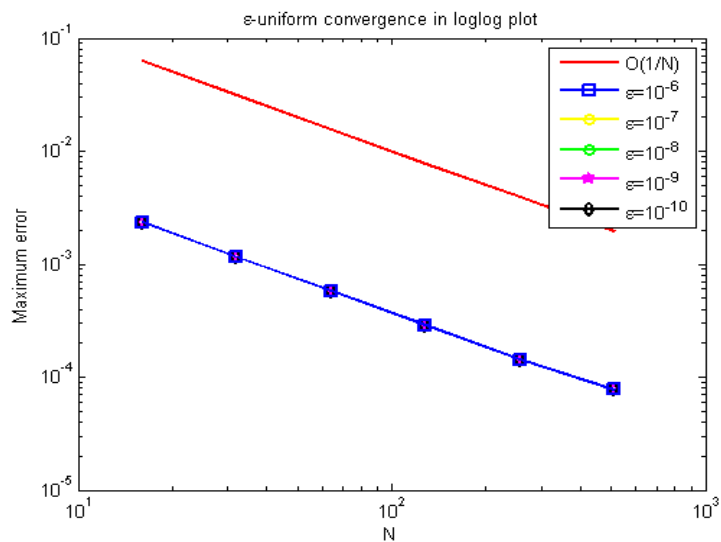

(a)

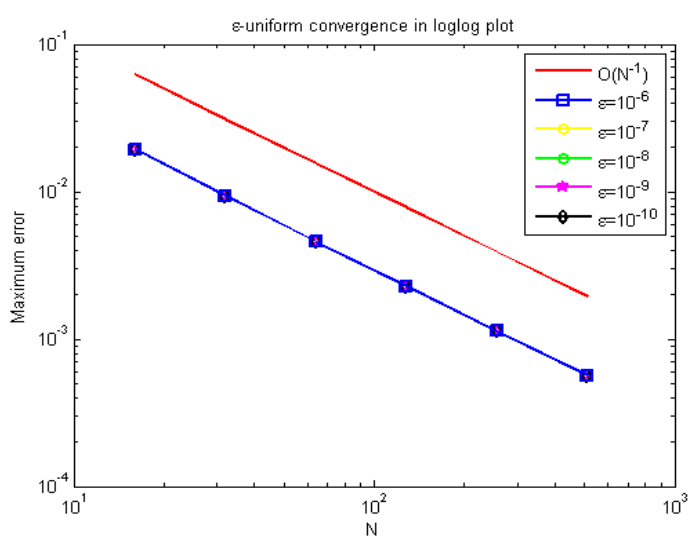

(b)

Figure. 3 The Log-Log plot of the maximum error for Example 1 on the left and Example 2 on the right side 
Table 3: Maximum absolute errors and rate of convergence for Example 2 at number of mesh points $N$ and $\Delta t$

\begin{tabular}{cccccc}
\hline$\varepsilon \downarrow$ & $N=16$ & $N=32$ & $N=64$ & $N=128$ & $N=256$ \\
& $\Delta t=0.1 / 2$ & $\Delta t=0.1 / 2^{2}$ & $\Delta t=0.1 / 2^{3}$ & $\Delta t=0.1 / 2^{4}$ & $\Delta t=0.1 / 2^{5}$ \\
\hline $10^{-6}$ & $2.9520 \mathrm{e}-02$ & $1.5980 \mathrm{e}-02$ & $8.3421 \mathrm{e}-03$ & $4.2681 \mathrm{e}-03$ & $2.1581 \mathrm{e}-03$ \\
$10^{-7}$ & $2.9520 \mathrm{e}-02$ & $1.5980 \mathrm{e}-02$ & $8.3421 \mathrm{e}-03$ & $4.2681 \mathrm{e}-03$ & $2.1581 \mathrm{e}-03$ \\
$10^{-8}$ & $2.9520 \mathrm{e}-02$ & $1.5980 \mathrm{e}-02$ & $8.3421 \mathrm{e}-03$ & $4.2681 \mathrm{e}-03$ & $2.1581 \mathrm{e}-03$ \\
$10^{-9}$ & $2.9520 \mathrm{e}-02$ & $1.5980 \mathrm{e}-02$ & $8.3421 \mathrm{e}-03$ & $4.2681 \mathrm{e}-03$ & $2.1581 \mathrm{e}-03$ \\
$10^{-10}$ & $2.9520 \mathrm{e}-02$ & $1.5980 \mathrm{e}-02$ & $8.3421 \mathrm{e}-03$ & $4.2681 \mathrm{e}-03$ & $2.1581 \mathrm{e}-03$ \\
\hline
\end{tabular}

Table 4: Comparison of Maximum absolute errors and rate of convergence for Example 2 at number of mesh points $N$ and $\Delta t$ with $T=2$, for $\mathcal{E} \in\left\{10^{-10}, 10^{-9}, \ldots, 10^{-6}\right\}$.

\begin{tabular}{|c|c|c|c|c|c|}
\hline & $\begin{array}{c}N=16 \\
\Delta t=0.1 / 2\end{array}$ & $\begin{array}{c}N=32 \\
\Delta t=0.1 / 2^{2}\end{array}$ & $\begin{array}{c}N=64 \\
\Delta t=0.1 / 2^{3}\end{array}$ & $\begin{array}{c}N=128 \\
\Delta t=0.1 / 2^{4}\end{array}$ & $\begin{array}{c}N=256 \\
\Delta t=0.1 / 2^{5}\end{array}$ \\
\hline \multicolumn{6}{|c|}{ Present method } \\
\hline$E^{N, \tau}$ & $2.9520 \mathrm{e}-02$ & $1.5980 \mathrm{e}-02$ & $8.3421 \mathrm{e}-03$ & $4.2681 \mathrm{e}-03$ & $2.1581 \mathrm{e}-03$ \\
\hline$r^{N, \tau}$ & 0.88543 & 0.93778 & 0.96682 & 0.98383 & 0.99180 \\
\hline \multicolumn{6}{|c|}{ Result in Elango et al., (2021) } \\
\hline$E^{N, \Delta t}$ & $1.8765 \mathrm{e}-01$ & $1.4776 \mathrm{e}-01$ & $9.7571 \mathrm{e}-02$ & $5.7092 \mathrm{e}-02$ & $3.1057 \mathrm{e}-02$ \\
\hline$r^{N, \Delta t}$ & 0.34479 & 0.59873 & 0.77316 & 0.87837 & 0.93697 \\
\hline
\end{tabular}

From Tables 1 and 3, it can be observed that the computed maximum point wise errors $E_{\varepsilon}^{N, \Delta t}$ after a certain value of $\varepsilon=10^{-6}$ (for both problems) are stable, and uniformly convergent. From Tables 2 and 4, the performance of the proposed scheme is investigated by comparing with recently published paper in Elango et al. (2021). As one sees, the proposed method gives more accurate results. To observe the changes in the boundary layer width with respect to $\mathcal{E}$, and to show the physical behavior of the solution, the surface plots of the numerical solution (Figs. 1 and 2) have been plotted. From the figures, for small $\mathcal{E}$ close to zero twin boundary layers at $x=0$ and $x=2$ further an interior layer at $x=1$ can be seen from the solution. The numerical solutions obtained by the present method have been log-log plotted for singular perturbation parameter ranging from $\varepsilon=10^{-6}$ to $10^{-10}$ in Figure (3) to indicate the maximum absolute errors decrease as the number of the mesh points increases and maximum absolute errors increases as the perturbation parameters decreases. This is one of the main results to be shown in this paper.

\section{Conclusions}

In this work, parameter uniform numerical method has been developed for solving singularly perturbed delay parabolic differential equation with non-local boundary condition exhibiting parabolic boundary layers and an interior layer. The developed method is based on the method of a line that constitutes the non-standard finite-difference (NSFDM) for the spatial discretization and a classical backward Euler method is used in the temporal direction for the system of initial value problem resulting from the spatial discretization. To treat the non-local boundary condition, Simpson's rule is applied. The stability and convergence of the proposed scheme are analyzed. Two model examples have been considered to validate the applicability of the scheme by taking different values for the perturbation parameter $\mathcal{E}$ and mesh points. The computational results are presented in terms of tables and figures. The proposed numerical scheme is shown to be accurate of order $O\left(h^{2}+\Delta t\right)$. And the performance of the proposed scheme is investigated by comparing with prior study; it also improves the results of the methods existing in the literature. 


\section{Acknowledgement}

The authors would like to thanks Jimma Universities for financial and material support.

\section{References}

Ansari A.R, Bakr S.A, Shishkin G.I, 2007. A parameter-robust finite difference method for singularly perturbed delay parabolic partial differential equations, Journal of Computational and Applied Mathematics Vol. 205, pp. 552-566. https://doi.org/10.1016/j.cam.2006.05.032

Ansari A. R., Hegarty. A. F., Shishkin. G.I., 2003. A note on fitted operator methods for the classical laminar free jet problem, Applied Numerical Mathematics,Vol.45, pp.353-365. https://doi.org/10.1016/S0168-9274(02)00225-8

Amiraliyev G., Amiraliyeva I., Kudu M., 2007.A numerical treatment for singularly perturbed differential equations with integral boundary condition, Applied Mathematics and Computation, Vol.185, No.1, pp. 574-582. https://doi.org/10.1016/j.amc.2006.07.060

Amiraliyev G.M, Ylmaz B. 2014. Finite difference method for singularly perturbed differential equations with integral boundary condition, International Journal of Mathematics and Computation, Vol.22, No1, pp.1-10.

Baker, C.T.H , Bocharov.G.A and Rihan.F.A, 1999. A report on the use of delay differential equations in numerical modelling in the biosciences, Department of Mathematics, University Manchester, Manchester, England.

Bansal K., Sharma K.K, 2018. Parameter-robust numerical scheme for time-dependent singularly perturbed reaction-diffusion problem with large delay, Numerical Functional Analysis and Optimization, Vol. 39, pp. 127-154. : http://dx.doi.org/10.1080/01630563.2016.1277742

Bahuguna D., Dabas, J.: 2008. Existence and uniqueness of a solution to a semilinear partial delay differential equation with an integral condition. Nonlinear Dynamics and Systems Theory, Vol. 8, No.1, pp. 7-19.

Bashier E.B.M, Patidar K.C.,2011a. A novel fitted operator finite difference method for a singularly perturbed delay parabolic partial differential equation, Applied Mathematics and Computation, Vol.217, pp.4728-4739. https://doi.org/10.1016/j.amc.2010.11.028

Bansal K., and Sharma K.K., 2017. Parameter uniform numerical scheme for time dependent singularly perturbed convectiondiffusion-reaction problems with general shift arguments, Numerical Algorithm, Vol.75, No. 1, pp. 113-145. DOI 10.1007/s11075-016-0199-3

Clavero. C, Jorge J.C., Lisbona F., Shishkin G.I.,, 2000. An alternating direction scheme on a non-uniform mesh for reactiondiffusion parabolic problems, IMA Journal of Numerical Analysis, Vol. 20, No 2, pp. 263-280. DOI: 10.1093/imanum/20.2.263

Daba.I and File G., 2020. Extended cubic B-spline collocation methods for singularly perturbed parabolic differential $\square$ difference equation arising in computational neuroscience, International Journal for Numerical Methods in Biomedical Engineering, e3418, https://dio.org/10.1002/cnm.3418

Debela G., and Duressa F., 2019. Exponentially fitted finite difference method for singularly perturbed delay differential equations with integral boundary condition, International Journal of Engineering \& Applied Sciences, Vol.11, No.4, pp.476-493. http://dx.doi.org/10.24107/ijeas.647640

Debela G., and Duressa F., 2020. Uniformly convergent numerical method for singularly perturbed convection-diffusion type problems with nonlocal boundary condition, International Journal for Numerical Methods in Fluids, pp.1-13. https://doi.org/10.1002/fld.4854

Debela G., and Duressa F., 2020. Accelerated fitted operator finite difference method for singularly perturbed delay differential equations with non-local boundary condition, Journal of the Egyptian Mathematical Society, Vol. 28, No. 16 https://doi.org/10.1186/s42787-020-00076-6

Elango et al., 2021. Finite difference scheme for singularly perturbed reaction diffusion problem of partial delay differential equation with nonlocal boundary condition, Advances in Difference Equations (2021), 2021:151 https://doi.org/10.1186/s13662021-03296-x

Kumar D., Kumari P., 2020. Parameter-uniform numerical treatment of singularly perturbed initial-boundary value problems with large delay, Applied Numerical Mathematics Vol.153, pp.412-429. https://doi.org/10.1016/j.apnum.2020.02.021

Kumar, D., Kadalbajoo, M.K.: 2011. A parameter-uniform numerical method for time-dependent singularly perturbed differentialdifference equations, Applied Mathematics and Model, Vol. 35, No. 6, pp. 2805-2819. https://doi.org/10.1016/j.apm.2010.11.074

Kuang Y. 1993. Delay Differential Equations: With Applications in Population Dynamics, Academic Press, Boston, San Diego, New York.

Kudu M., Amiraliyev G., 2015. Finite difference method for singularly perturbed differential equations with integral boundary condition, International Journal of Mathematics and Computation, Vol. 26, No3, pp.71-79.

Ladyzhenskaya O.A., Solonnikov V.A., Ural'tseva N.N, 1968. Linear and quasi-linear equations of parabolic type, Translations of Mathematical Monographs,vol. 23, American Mathematical Society, USA Mahaffy.P.R., A'Hearn.M.F, Atreya S.Ket.al, 1999. The Champollion cometary molecular analysis experiment, Advances in Space Research Vol. 23No.2, pp. 349-359. 
Miller J.J, O’Riordan E, Shishkin G., 1996. Fitted Numerical Methods for Singular Perturbation Problems: Error Estimates in the Maximum Norm for Linear Problems in One and Two Dimensions, World Scientific. https://doi.org/10.1142/8410

Miller J.J.H, O’Riordan E., Shishkin G.I., Shishkina L.P, 1998. Fitted mesh methods for problems with parabolic boundary layers, Mathematical Proceedings Royal Irish Academy, Vol. 98A, No.2, pp. 173-190.

Mickens R.E., 2005.Advances in the Applications of Nonstandard Finite Difference Schemes, World Scientific, Singapore, New Jersey, London, Hong Kong.

Mekonnen T.B., File G. 2021. Uniformly convergent numerical method for two-parametric singularly perturbed parabolic convection-diffusion problems, Journal of Applied and Computational Mechanics, Vol. 7, No2,pp. 535-545. DOI: 10.22055/JACM.2020.35193.2596

Patidar K.C, Sharma K.K, 2006.Uniformly convergent nonstandard finite difference methods for singularly perturbed differential difference equations with delay and advance, International Journal of Numerical Methods and Engineering, Vol. 66, No. 2, pp.272-296. DOI: 10.1002/nme.1555

Roos, H.G., Stynes M., and Tobiska L., 2008. Robust Numerical Methods for Singularly Perturbed Dierential Equations: Convection-Diusion-Reaction and Flow Problems. Springer Science and Business Media, Vol. 24, Berlin Heidelberg.

Singh, J.,Kumar, S., and Kumar, M. 2018. A domain decomposition method for solving singularly perturbed parabolic reactiondiffusion problems with time delay. Numerical Methods for Partial Differential Equations, Vol.34, No.5, pp. 1849-1866. https://doi.org/10.1002/num.22256

Sekar E, Tamilselvan A., 2019. Singularly perturbed delay differential equations of convection-diffusion type with integral boundary condition, Journal of Applied Mathematics and Computing Vol. 59, No. (1-2), pp. 701-722. https://doi.org/10.1007/s12190-018-1198-4

Tesfaye A, Gemechis F., Guy A, 2021. Robust finite difference method for singularly perturbed two-parameter parabolic convection-diffusion problems, International Journal of Computational Methods, Vol. 18, No. 2, 2050034. DOI: 10.1142/S0219876220500346

Woldaregay M .and Duressa F., 2022. Uniformly convergent numerical method for singularly perturbed delay parabolic differential equations arising in computational neuroscience, Kragujevac Journal of Mathematics, Vol. 46, No.1, pp.65-84, Article in press

\section{Biographical notes}

Wakjira Tolassa Gobena received BSc from Addis Ababa University in Mathematics MSc in mathematics (Numerical Analysis) from Jimma University, Ethiopia in 2010 and 2016, respectively. Currently he is a PhD candidate at Jimma University, Jimma, Ethiopia.

Dr. Gemechis File Duressa is an Associate Professor of Mathematics at Jimma University, Jimma, Ethiopia. He has many years of teaching experience at the university level. Currently he is working as dean of college of Natural sciences in Jimma University. He has more than 71publications 